\title{
On the epistemological foundations of the law of the lever
}

\section{Maarten Van Dyck}

Department of Philosophy and Moral Sciences, Ghent University, Blandijnberg 2, B-9000 Gent, Belgium; maarten.vandyck@ugent.be

\begin{abstract}
In this paper I challenge Paolo Palmieri's reading of the Mach-Vailati debate on Archimedes's proof of the law of the lever. I argue that the actual import of the debate concerns the possible epistemic (as opposed to merely pragmatic) role of mathematical arguments in empirical physics, and that construed in this light Vailati carries the upper hand. This claim is defended by showing that Archimedes's proof of the law of the lever is not a way of appealing to a non-empirical source of information, but a way of explicating the mathematical structure that can represent the empirical information at our disposal in the most general way.
\end{abstract}

\section{Keywords}

Ernst Mach

Giovanni Vailati

Archimedes

Law of the lever

Mathematical physics

\section{Introduction}

In an interesting recent article, Paolo Palmieri claims to resolve the one century old debate between Ernst Mach and Giovanni Vailati on Archimedes's proof of the law of the lever. ${ }^{1} \mathrm{He}$ claims that Vailati was right in pointing out that Mach's criticism of this proof was historically inaccurate, but that Mach's epistemological point about the proof remains valid. I

\footnotetext{
${ }^{1}$ Palmieri (2008).
} 
will argue that, on the contrary, Vailati's case still stands, also against Palmieri's sophisticated counter-arguments.

However, the motivation for writing this reply in no way comes from a desire to pass judgements on who is right and who is wrong. I rather think that Palmieri's sympathetic defence of Mach offers us as an excellent occasion to come to a more precise understanding of some of the issues that were at stake in the debate (issues which Palmieri also explicitly wants to put back on the agenda). So I hope that by pressing Vailati's case against Palmieri we can use this dialectical exchange to come to a firmer grip on what we are exactly targeting when debating the epistemological foundations of a law in mathematical physics, such as the law of the lever.

In Section 2, I will quickly summarize Palmieri's reading of the Mach-Vailati debate, and introduce the bare essentials of one of his counterarguments against Vailati. In Section 3, I will return to Vailati's original paper and try to indicate why Palmieri's argument fails as a counter-argument. I will then use this in Section 4 to reformulate what exactly Vailati was opposing to Mach. In Section 5, I will further defend Vailati by taking another look at Archimedes's proof of the law of the lever; and in the concluding Section 6, I will then use this analysis to assess the second argument that Palmieri gives in his paper. I will argue that while in substance right, it can actually be used to show why Vailati offers a much more fruitful view on what is going on in Archimedes's proof of the law of the lever than Mach does.

\section{Palmieri's argument in favour of Mach}

Both the spirit and content of Mach's criticism are best captured by his well-known rhetorical question how it would be possible to derive "by speculative methods" the inverse proportionality of weight and lever-arm "from the mere assumption of the equilibrium of equal weights at equal distances". ${ }^{2}$ Mach's answer is of course that this is impossible, and that Archimedes's rational argument is defective as it actually presupposes what needs to be proved. He moreover locates the exact step in the argument where this happens, in what Palmieri calls the equilibrium-preserving assumption. This assumption basically states that equilibrium is not disturbed if one of the equilibrating weights is replaced with two weights

\footnotetext{
${ }^{2}$ Mach (2007 [1902], p. 14.
} 
placed symmetrically around the original weight, each having half the weight of the original body.

Palmieri accepts Vailati's historical correction to Mach's analysis that Archimedes actually could justify this assumption by appealing to the properties of a body's centre of gravity, which he in all probability treated in an earlier lost treatise; and that it is thus historically inaccurate to claim that this step must have been made by covertly appealing to the law of the lever. ${ }^{3}$ Nonetheless, he also claims that this does not diminish the philosophical point made by Mach, which he constructs as the question whether the principles of equilibrium "have an empirical origin, or an essentially logical, that is, self-evident character". ${ }^{4}$ To sharpen this question he also introduces the more general counter-argument that Vailati made against Mach's general claim, which he then shows to be defective in exactly the way he thinks Mach already warned us with respect to Archimedes's original proof.

Vailati's main point, as interpreted by Palmieri, is that the law of the lever actually "considers a larger class of displacements of weights", a class "that includes the class considered by the equilibrium-preserving assumption", and that the actual presupposition that enters into the law of the lever is accordingly weaker than its conclusion. It seems to follow that by enlarging the class of valid replacements Archimedes "would succeed in showing that, from what he assumes as self-evident premises, a conclusion can be derived which is valid for a vaster class of phenomena which are not self-evident". 5

To see that this cannot be the case Palmieri proposes to analyze the mathematical formulation that Vailati had given to his counterargument. On the one hand we have the equilibriumpreserving assumption, which can be expressed as follows ( $p$ expressing the weight, $d$ the distance from the fulcrum and $h$ the equal distances from $d$ at which we place the divided weights, and $F$ an unspecified function of distance):

$$
p \cdot F(d)=p / 2 \cdot F(d+h)+p / 2 \cdot F(d-h)
$$

On the other hand we have the law of the lever, stating that two weights $p_{1}$ and $p_{2}$ are in equilibrium with another weight $p$ on the opposite side of the lever if:

\footnotetext{
${ }^{3}$ Vailati's original paper of 1904 is reprinted in Vailati (1987).

${ }^{4}$ Palmieri (2008), p. 43.

${ }^{5}$ Palmieri (2008), p. 49.
} 


$$
p \cdot d=p_{1} \cdot d_{1}+p_{2} \cdot d_{2}
$$

We can see that whereas (1) is restricted to replacements which are symmetric with respect to the original situation, (2) sanctions a much larger class of also non-symmetric replacements. But is it really the case that (2) allows us to broaden the class of replacements? Palmieri gives an ingenious argument (the details of which need not concern us here) that this cannot be the case, by establishing that there is actually only one unique monotonically increasing function $F(d)$ satisfying (1), and that this is the function $F(d)=\mathrm{K} d$ (with $\mathrm{K}$ a positive real constant). ${ }^{6}$ So it seems that Vailati's counter-argument, notwithstanding its at first sight intuitive appeal, is actually plagued by the same kind of "self-deception" that Mach had already diagnosed in Archimedes's proof. It is only an illusion that we can find out something new about equilibrium by purely rational means - all replacements which are allowed by (2) are already allowed by (1).

\section{But what was Vailati up to?}

It is clear that Palmieri attributes to Vailati the view that the step from (1) (the equilibriumpreserving assumption) to (2) (the law of the lever) is of some kind of an ampliative nature (where I take "ampliative" as the general term for arguments having conclusions that go beyond the premises). But it is not at all clear whether this is really Vailati's opinion. It is true that Vailati's wording is rather ambiguous, as noted by Palmieri in a footnote where he comments on the problem of interpreting the original Italian, which states "la premessa, su cui si basa il ragionamento d'Archimede asserisce solo una parte della conclusione generale a cui egli arriva partendo da essa". ${ }^{7}$ As Palmieri points out: "premises do no assert conclusions. Rather a conclusion is derived from premises by some inference", and he concludes "the meaning of Valiati's passage is perhaps that the premises somehow contain only a portion of the conclusion". But I think it is extremely doubtful that Vailati thought that the conclusion would contain more empirical information, and that the law of the lever would thus have an extra-empirical source of information somehow coming from the supposedly self-evident

\footnotetext{
${ }^{6}$ Cf. Palmieri (2008), p. 50, for the precise formulation of what he calls the "Vailati constraint" and the mathematical argument.

${ }^{7}$ Vailati (1987), p. 221. Cf. Palmieri (2008), p. 49, fn. 13.
} 
steps in Archimedes's argument. To see this, let us quote from the conclusion of Vailati's paper:

The assertion that Archimedes's premises already implicitly contain the conclusion that he deduces from them, seems to be true to me only in the sense in which it is true of any demonstration in the mathematical sciences, insofar that in each of these the truth of the propositions that are demonstrated appears as a simple consequence of opportune operations of choice, of concatenation, of coordination following on the fundamental propositions that are put at the basis of the treatment. ${ }^{8}$

This leaves us wondering in what way Palmieri actually has provided a counter-argument to Vailati, when what he has actually done is to give a new kind of mathematical argument showing that (2) is mathematically implied by (1). Vailati's point rather seems to have been that the argument leading from (1) to (2), although strictly speaking not adding empirical content is still informative - exactly in the way that any relevant mathematical demonstration is that makes explicit what was contained merely "implicitly" in a set of premises. 9

So, is it the case that (2) allows us to broaden the class of replacements? Not in the sense that empirical information is added, but that was not Vailati's point. His point rather was that this of course need not imply that the empirical content contained in (1) cannot be formulated in a more interesting way as the consequence of "opportune" mathematical manipulations. And it appears that (2) is indeed a more informative way to describe what is allowed by (1). It seems to me that this is why Vailati states a bit awkwardly that the premises only assert part of the conclusion.

\section{On mathematical arguments in empirical physics}

Palmieri thinks that Vailati's argument is a way of appealing to some extra, presumably rational ("self-evident") source of information. We have now seen that this does not seem to have been the way Vailati understood his own argument. But we can still wonder in what way we can actually call one proposition derived from another through mathematical manipulations more "informative" than the other. This also seems to have been Mach's

\footnotetext{
${ }^{8}$ Vailati (1987), p. 225.

${ }^{9}$ Dijksterhuis criticized Mach in very similar terms; cf. Dijksterhuis (1987 [1953]), p. 294.
} 
reaction to Vailati's reply (although the terminology of informativeness is mine and not explicitly found in Vailati or Mach). ${ }^{10}$ He concedes the point that Archimedes may have used the equilibrium-preserving assumption based on an (implicit) appeal to propositions describing the properties of a body's centre of gravity, rather than on a viciously circular appeal to the law of the lever itself. But he stresses that this does not vitiate his crucial claim (a view in which he is now followed by Palmieri), because these propositions in their turn must already contain the same empirical information as the law of the lever: if the demonstration is not circular, it is thus at least "artificial and roundabout" (as it does not actually add anything substantial). It follows that its only possible worth must lie in its capability to "surprise" the reader, but that it is completely impotent to add any "insight". 11 The "informativeness" that can be claimed must thus be purely pragmatic - the demonstration shows a fact that is surprising to the uneducated reader, but this has no real epistemic worth. We can thus see that what was really at stake in the exchange between Vailati and Mach is the epistemic role of mathematical deduction in empirical physics. By focussing on the opposition between the empirical and the self-evident dimensions of mechanics, Mach seems to downplay its role (and he is followed in this respect by Palmieri), whereas Vailati's point is exactly that this opposition is maybe too crude to do justice to the workings of mathematical physics.

According to Mach, Archimedes's derivation of the law of the lever must have been a case of "self-deception" (the deduction must have been "defective", or, at best, "artificial"), because being of a purely mathematical nature it cannot add anything of worth to what is already known - scientific propositions are either empirical or self-evident, and since the law of the lever is clearly not self-evident, it must be empirical; so the only kind of valid argument in favour of it must be of an essentially empirical kind, i.e. supposedly direct (inductive) confirmation of its truth.

Vailati, on the contrary, can be read as trying to indicate that there is an alternative way to understand what is going on: rather than having to appeal to a direct confirmation of the proportionality stated in the law of the lever, we can see Archimedes as using mathematics to reorganize the empirical information already at his disposal (as expressed in the equilibriumpreserving assumption) with as gain the important new insight that this information is enough

\footnotetext{
${ }^{10}$ Mach's reaction was included in the seventh German edition of his work, and can be found in Mach (2007 [1915]), pp. 1-3.

${ }^{11}$ Mach (2007 [1915]), pp. 2-3.
} 
to underwrite the validity of the precise mathematical law for the equilibrium of a balance. It is accordingly not surprising to find him pointing out that proving the law of the lever in the way that Archimedes does, gives one a possible way to recognize the truth of this mathematical law without having to establish it inductively through direct measurement. ${ }^{12}$

\section{Proving the law of the lever}

To make more precise the point of view attributed here to Vailati, let us have another look at Archimedes's proof of the law of the lever (we'll restrict attention to proposition 6, i.e. the commensurable case), and let us especially take serious Vailati's suggestion that the equilibrium-preserving assumption is introduced as following from the properties of any body's centre of gravity.

Archimedes's proof proceeds by considering two bodies on a balance that satisfy the conditions for the law of the lever (i.e. weights inversely as distances from the fulcrum), and showing that they must be in equilibrium. ${ }^{13}$ What actually needs to be proven, Archimedes asserts, is that the centre of gravity of the magnitude composed from these two bodies must coincide with the fulcrum. To prove that this is the case, he first shows that, for purely geometrical reasons, it is possible to divide the two bodies in smaller equal weights which can be distributed along the lever arms in a certain configuration. In a second step he then appeals to the earlier proven proposition 5 to conclude that in this configuration the common centre of gravity of the parts which made up the first body coincides with the position of the original body's centre of gravity, and similarly for the second body. Moreover, because all these parts taken together have their common centre of gravity coinciding with the fulcrum, they are in equilibrium. It follows that also the two original bodies must be in equilibrium.

It is clear that Archimedes's proof presupposes some properties of a body's centre of gravity: in the first place, the identification of what needs to be proven as the coincidence of the common centre of gravity of the two bodies with the fulcrum assumes that suspension in the centre of gravity is a sufficient reason for equilibrium; ${ }^{14}$ in the second place, the assumption

\footnotetext{
${ }^{12}$ Vailati (1987), p. 224.

${ }^{13}$ Archimedes (1544), p. 127

${ }^{14}$ This follows e.g. from the definition of centre of gravity as given by Pappus in the eight book of his mathematical collections. Renaissance commentators such as Guidobaldo del Monte thus reasonably assumed that the only correct way to understand Archimedes was to supplement his treatise with this definition (Del Monte (1588), p. 8).
} 
that bodies of equal weight with coinciding centres of gravity (such as one of the original bodies and the body composed of its parts distributed over the lever arms) can be substituted for each other without changing equilibrium. ${ }^{15}$ The latter supposition (which as Vailati points out comes down to assuming a body's centre of gravity to be unique) ${ }^{16}$ of course provides the physical underpinning for the "equilibrium-preserving assumption"; this connection is further strengthened by the fact that this supposition is actually only used for the case where a body is replaced by a number of bodies placed symmetrically around its original position.

But we must be careful in interpreting what this restriction implies. The general supposition concerning the replacement of bodies with equal weights and coinciding centres of gravity is only used in its restricted form (equivalent with (1)) because only for this symmetrical configuration can we already determine the centre of gravity of the split bodies - and we can do this because of Archimedes's postulate that equal bodies at unequal distances are not in equilibrium (which implies that the only possible position for their unique common centre of gravity is the middle point between the two bodies, as proven in proposition 4). We thus have a general replacement property which we at first can only apply in the limited case of symmetrical replacements; and Archimedes's proof now shows that, for purely geometrical reasons, this is enough to determine the common centre of gravity of any two bodies - and thus to apply the replacement property in its full scope, which can now be mathematically expressed by (2).

Just as in geometry we can prove properties valid for polygons on the basis of considerations of the properties of triangles, so Archimedes shows that we can prove properties of equilibrium in general on the basis of considerations of the properties of a special case of equilibrium, Valiati comments ${ }^{17}$ - the reason apparently being that just as we can construe all polygons from triangles, so we can transform the two cases of equilibrium in each other (because of the general replacement property as applied to symmetrical replacements and some further general geometrical relations that hold when the conditions for the law of the lever are met).

Rather than seeing the move from (1) to (2) as adding empirical content (as Palmieri construes it), it must be seen as adding mathematical determination (what I take to be Vailati's original

\footnotetext{
${ }^{15}$ Dijksterhuis (1987) has argued that this is actually postulated in postulate 6. Guidobaldo Del Monte also comments extensively on the use of this presupposition, the validity of which he thinks follows from Pappus's definition of centre of gravity (Del Monte (1588), passim).

${ }^{16}$ Vailati (1987), pp. 222-223.

${ }^{17}$ Vailati (1987), p. 225.
} 
position). We start from some very general properties of a centre of gravity (its uniqueness and its link with equilibrium) and some qualitative facts about equilibrium of weights (such as that equal weights at unequal distance are not in equilibrium), and Archimedes's proof then allows us to see how this is enough to determine the exact mathematical position of any two bodies' common centre of gravity. Even if the presuppositions already ("implicitly") contain all relevant empirical information, it is undeniable that it is only with the formulation of the law of the lever that we have explicated the mathematical structure that can represent the empirical information at our disposal in the most general way - because it is on its basis that we can now mathematically determine the centre of gravity of any system of weights.

We can conclude with Vailati against Mach and Palmieri that there is nothing mysterious about the fact we can, "by speculative methods ... discover the form of this dependence, the proportionality" - that is simply what mathematics allows us to do, once we have gathered the right kind of qualitative information!

\section{The limited validity of the law of the lever}

Palmieri's analysis of the mathematical equivalence between (1) and (2) is however only part of a two-pronged attack destined to decide the question of the empirical vs. self-evident nature of the rules of equilibrium. In what follows I will argue that the second prong of his attack brings an important fact to the fore, all too often overlooked in debates on the epistemological foundations of the law of the lever; but that it is only from the perspective sketched in the previous section that it can be fully appreciated. So I claim that Palmieri's further argument actually speaks in favour of Vailati, and ironically (given the author's own intentions) shows the unsatisfactory nature of the Machian position.

In the longest section of his paper Palmieri rehearses some features of the composition of forces in constant and non-constant gravity fields to remind us of the fact that the Archimedean law of the lever is at best an approximation to the true law holding for real-life ("non-Archimedean") balances on our earth (because of the fact that the forces of weight are actually due to a gravity field directed towards one central point - whereas the Archimedean law is strictly true only for parallel forces of weight). It is moreover not hard to show that for these real-life balances the equilibrium-preserving assumption also does not hold. Palmieri thus concludes that it is impossible, as a matter of fact, that the law of the lever could have its foundations in some purely speculative knowledge, as it is not even true! 
But this seems to leave us in a quandary: if we were to agree with Mach that the law of the lever should be underwritten by direct inductive evidence, how could we then ever have found out this merely approximate character (it is after all a very good approximation)?

However, if we take seriously the analysis offered in the previous section it is perfectly understandable how we could have come to such an insight. Archimedes's mathematical proof of the lever makes immediately clear what its conditions of validity are: the existence of the properties of centre of gravity as enumerated, among which its uniqueness in any body (which, remember, underwrites the possibility of the supposedly equilibrium-preserving replacements). It is accordingly not surprising that such diligent sixteenth century mathematicians as Guidobaldo del Monte and Simon Stevin already paid special attention to this assumption of uniqueness. Both pointed out that the convergence of weights in the centre of the world would imply that the centre of gravity of bodies on a balance would no longer be unique (as the effective component of the forces of weight would vary with the inclination of the balance), and thus would threaten the foundations of mechanics (Guidobaldo) or would make mechanics much more complicated as the correct law of the lever has to take on a more complicated form (Stevin). ${ }^{18}$

I would like to suggest that it is here that we find the true epistemic worth of mathematical demonstrations, such as Archimedes's, that are directed towards organizing empirical physics on a (semi-)axiomatic basis. This worth need not lie so much in the presumed self-evidence of the axioms (after all, both Guidobaldo and Stevin showed themselves perfectly capable of considering the possible denial of the uniqueness of a body's centre of gravity) - which issue I take to be somewhat of a red herring in the Mach-Vailati debate - but in the kind of tighter embedding it gives to the empirical claims. Showing in what way the validity of the law of the lever depends on some general and qualitative empirical properties of a body's centre of gravity gives you a way to learn some things about this law which you would otherwise be in no position to find out, such as the fact that Archimedes's law actually cannot be exactly true. We can conclude that Archimedes's mathematical deduction is indeed truly informative, and that there is nothing particularly "roundabout" or "artificial" about it - the latter judgement merely showing in what way Mach's opposition between the empirical and the self-evident

\footnotetext{
${ }^{18}$ Del Monte (1577), pp. 19v-20v (Guidobaldo's argument is analyzed in some detail in Van Dyck (2006)); Stevin (1955), pp. 112-115.
} 
leaves him without any satisfactory view on the role of mathematics in structuring our empirical knowledge. ${ }^{19}$

To sum up: I agree with Palmieri that the epistemological foundations of our knowledge of equilibrium (and certainly of all of physics) ultimately must be found in empirical facts; but I want to stress, with Vailati, that there is an indispensable role for mathematics in the way we build our sciences on these foundations, and that a Machian focus on the stark opposition between empirical and self-evident propositions threatens to blind us for this role.

\section{Acknowledgement:}

The author is Postdoctoral Fellow of the Research Foundation - Flanders (FWO).

\section{References}

Archimedes (1544). Opera. Basel: Ioannes Hervagius.

Del Monte, G. (1588). In duos Archimedis aequoponderantium libros paraphrasis sholijs illustrata. Pesaro: Apud Hieronymum Concordiam.

Dijksterhuis, E.J. (1987). Archimedes. (C. Dikshoorn, transl.). Princeton: Princeton University Press. (First published 1953.)

Mach, E. (2007). The science of mechanics: A critical and historical account of its development. (T.J. McCormack, transl.; $2^{\text {nd }}$ English edition). Merchant Books. (Original German edition published in 1902.)

Mach, E. (2007). The science of mechanics: A critical and historical account of its development. Supplementary Volume. (Containing the author's additions to the seventh German edition; P.E.B. Jourdain, transl.; $3^{\text {rd }}$ English edition). Merchant Books. (Original German edition published in 1915).

Palmieri, P. (2008). The empirical basis of equilibrium: Mach, Vailati, and the lever. Studies in History and Philosophy of Science, 39, 42-53.

Stevin, S. (1955). The principal works. Vol. 1. Mechanics. (E.J. Dijksterhuis, ed.). Amsterdam: Swets \& Zeitlinger.

\footnotetext{
${ }^{19}$ Let me add the caveat that this is a judgement on Mach's views as he expounds them while criticizing Archimedes's proof - it lies entirely beyond the ambitions of this short reply to try to assess in what way this could be expanded into a general criticism of his epistemological views.
} 
Vailati, G. (1987). Scritti. (M. Quaranta, ed.) (3 Vols.). Sala Bolognese: Forni Editori.

Van Dyck, M. (2006). Gravitating towards stability: Guidobaldo's Aristotelian-Archimedean synthesis. History of Sceince, 44, 373-407. 